\title{
Recurrent Infection and Immune Dysfunction: A Case of NCF-2 Gene Mutation with Secondary Hemophagocytic Lymphohistiocytosis
}

\author{
Fahad Fayyaz Butt ${ }^{a}$ Fatima Farid Mir ${ }^{a} \quad$ Anjan Madasu $^{b} \quad$ Hani Humad $^{b}$ \\ Asim Noor Ranab \\ ${ }^{a}$ General Pediatrics, Dubai Health Authority, Dubai, United Arab Emirates; ${ }^{b}$ Pediatric Hematology and Oncology \\ Department, Dubai Hospital, Dubai, United Arab Emirates
}

\section{Keywords}

Chronic granulomatous disease $\cdot$ NCF-2 gene mutation · Immunodeficiency - Recurrent infections .

Secondary hemophagocytic lymphohistiocytosis

\begin{abstract}
A 3-month-old female was referred to our facility for evaluation of recurrent severe infections. Her medical history was rich with frequent hospital admissions for intravenous antibiotic courses, blood transfusions on two separate occasions, and a recent growth of Pseudomonas aeruginosa on bronchoalveolar lavage culture. During inpatient stay, culture-sensitive antibiotics, broad-spectrum antifungals, and blood products were administered. Her blood, urine, and spinal fluid cultures, as well as serum/respiratory viral screening panels, yielded nil. In view of persistent high-grade fever, raised inflammatory markers, and recurrent infections, whole-exome sequencing was performed with the suspicion of an underlying immunodeficiency syndrome and/or primary hemophagocytic lymphohistiocytosis (HLH). A homozygous pathogenic variant c.229C >T(p.R77X) in the NCF-2 (neutrophil cytosolic factor 2) gene was detected, with no concurrent primary HLH mutations. Thus, a diagnosis of chronic granulomatous disease with secondary HLH was confirmed. Aggressive continuation of antimicrobial therapy soon led to defervescence, recession of inflammatory
\end{abstract}

karger@karger.com www.karger.com/dmj

Karger"
(C) 2022 The Author(s)

Published by S. Karger AG, Basel

This is an Open Access article licensed under the Creative Common Attribution-NonCommercial-4.0 International License (CC BY-NC) (http://www.karger.com/Services/OpenAccessLicense), applicable to the online version of the article only. Usage and distribution for commercial purposes requires written permission. markers, and improvement in general wellbeing. Notably, the institution of definitive $\mathrm{HLH}$ treatment protocol was not required as the triggering element was targeted. The patient was then repatriated on prophylactic antibiotics with strong recommendation for bone marrow transplantation.

(C) 2022 The Author(s)

Published by S. Karger AG, Basel

\section{Introduction}

Chronic granulomatous disease (CGD) is a rare primary disorder of NADPH oxidase complex components resulting in defective superoxide burst and intracellular phagocytic killing [1]. Incidence is 1 in 250,000 cases, with $70 \%$ due to X-linked recessive inheritance of a CYBB gene mutation and the remainder from autosomal mutations in NCF1 (20\%), CYBA (5\%), NCF2 (5\%), or NCF4 (1 individual) [2]. The majority of cases are diagnosed earlier than 5 years of age with unusually persistent lymph node and skin abscesses, pneumonia, osteomyelitis, liver abscesses, BCG site infections, and colitis, commonly alongside positive family histories of early death [3-9]. HLH presents in two forms, of which secondary cases betide repeated infection, autoimmune disease, and malignancy [10]. Where children with CGD suffer substantial recurrent infections, the forthcoming risk for secondary HLH is significant. Our report exhibits the clinical course 
of previously undiagnosed CGD complicated by secondary $\mathrm{HLH}$, the latter of which was amenable to aggressive antimicrobial therapy alone.

\section{Case Report}

A 3-month-old female was referred to our facility for treatment and further evaluation of recurrent severe infections. She was born at term via normal vaginal delivery to first cousin consanguineous parents. The antenatal period was remarkable for gestational diabetes controlled on insulin. Her birth weight was $2.5 \mathrm{~kg}$ and postnatally had required phototherapy for Rh- incompatibility-induced neonatal jaundice. She was exclusively breast-fed since birth and received routine immunizations. The family history was remarkable for two second trimester spontaneous miscarriages prior to her birth.

The patient was active and thriving until her twenty-fourth day of life, when she developed bronchopneumonia requiring a 5-day inpatient course of intravenous (IV) antibiotics. Within a week of discharge, she was re-admitted with fever under the suspicion of neonatal sepsis. Full septic workup was sterile, and once again she was discharged home after an IV antibiotic course. By the second month of life, the child developed right-sided pneumonia and sepsis requiring the third inpatient admission for approximately 2 weeks. Screening for tuberculosis, cytomegalovirus serology, and immunoglobulin levels was all unremarkable. At the age of 3 months, the patient was re-admitted with severe bronchopneumonia, necessitating computed tomography (CT) of the chest with contrast and diagnostic bronchoscopic evaluation. The CT features depicted bilateral pneumonia with early necrotic changes, and bronchoalveolar lavage grew Pseudomonas aeruginosa. Blood and urine cultures, COVID-19 nasal reverse transcriptase polymerase chain reaction test, sweat chloride testing, and screening echocardiography were unremarkable. At this junction, unlike her previous admissions, the infant displayed persistence of fever despite over 2 weeks of culture-sensitive antibiotic therapy, whereupon she was transferred to our tertiary hospital.

On presentation to our facility, the child was tachypneic, maintaining oxygen saturation on room air, temperature $38^{\circ} \mathrm{C}$, heart rate 160 beats/min, blood pressure $75 / 52 \mathrm{~mm} \mathrm{Hg}$, and capillary refill under $2 \mathrm{~s}$. She was nondysmorphic, well hydrated, and well perfused. Growth parameters as per the WHO chart demonstrated her weight on 0.5 th percentile, length on 5th percentile, and head circumference on 3rd centile. Skin revealed a generalized maculopapular rash over trunk and perineum, with excoriation over the latter. Bilateral tender parotid swellings extending to the submandibular region were apparent. Chest was clear with no added sounds. Heart sounds were normal with a soft systolic murmur appreciated. The abdomen was distended with an everted umbilicus and significant hepatosplenomegaly. A complete septic workup was performed, following which IV meropenem and clindamycin were initiated. Laboratory findings were of mild bicytopenia (hemoglobin $9.2 \mathrm{~g} / \mathrm{dL}$ [reference range 11-14 $\mathrm{g} / \mathrm{dL}$ ] and platelets $187,000 / \mu \mathrm{L}$ [reference range 200,000-550,000/ $\mathrm{L}$ ]; white cell count $12,100 / \mu \mathrm{L}$ [reference range $6,000-18,000 / \mu \mathrm{L}]$ ), highly raised inflammatory markers (c-reactive protein $149 \mathrm{mg} / \mathrm{L}$ [reference range $<5 \mathrm{mg} / \mathrm{L}$ ]; procalcitonin $11.5 \mathrm{ng} / \mathrm{mL}$ [reference range $<0.05 \mathrm{ng} /$ $m L]$ ), positive growth of Staphylococcus hominis on peripheral

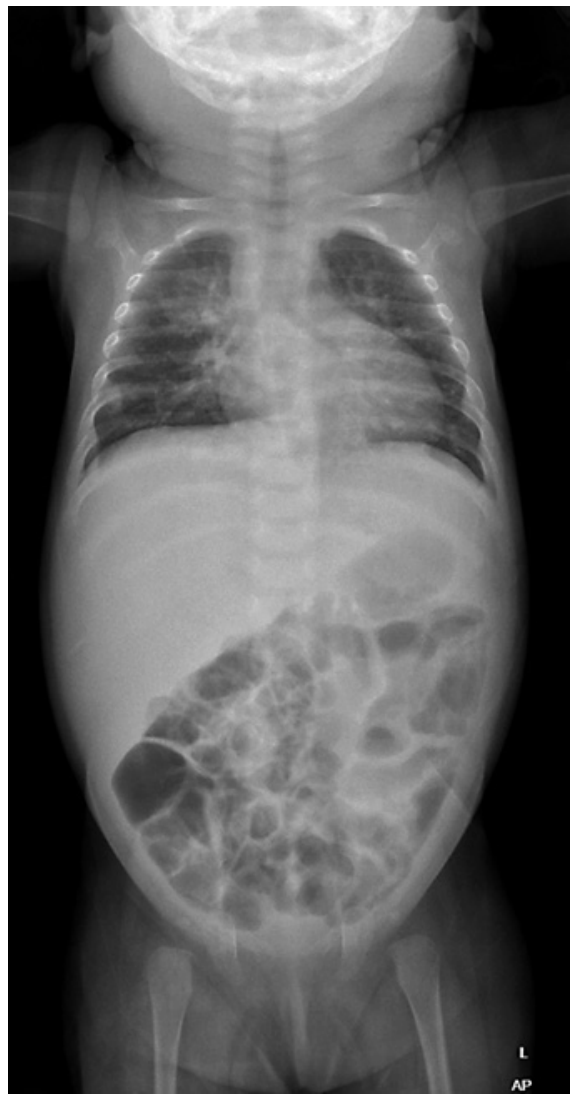

Fig. 1. Chest-abdomen X-ray with eleven ribs bilaterally, right upper lobe consolidation, and hepatomegaly.

blood culture, and sterile urine/spinal fluid cultures. Blood film demonstrated mild neutrophilia with toxic granulation and normocytic normochromic red cells with mild thrombocytopenia. Imaging wise, her chest-abdomen $\mathrm{X}$-ray revealed eleven ribs bilaterally, a right upper lobe consolidation, and hepatomegaly (Fig. 1), and ultrasound of the swollen neck and swollen parotid glands revealed features of bilateral cervical lymphadenopathy and left parotitis.

Extensive further investigations were sought in order to ascertain the etiology of persistent fever, parotitis, skin rash, hepatosplenomegaly, and bicytopenia. HIV, hepatitis B, Epstein-Barr virus, toxoplasmosis, herpes simplex I and II, and coxsackie virus serology testing yielded only coxsackie virus IgM positive. Cytomegalovirus DNA PCR was negative, as was the serum Aspergillus antigen level. Fluconazole was empirically initiated alongside antibiotics in view of high-grade fever and growing suspicion of an underlying immunodeficient state. Baseline immune status screening revealed normal serum immunoglobulin and lymphocyte subset counts. Our hospital did not host the facilities for dihydrorhodamine or nitroblue tetrazolium testing. The concurrence of HLH could not be undermined, hence investigated as well - revealing elevated levels of ferritin $(1,166 \mathrm{ng} / \mathrm{mL}$ [reference range $61-405 \mathrm{ng} / \mathrm{mL}$ ]), triglycerides (358 $\mathrm{mg} / \mathrm{dL}$ [reference range $82-258 \mathrm{mg} / \mathrm{dL}$ ]), lactate dehydrogenase (488 U/L [reference range $180-435 \mathrm{U} / \mathrm{L}$ ]), D-dimer $(4.11 \mu \mathrm{g} / \mathrm{mL}$ [reference range 
$<0.5 \mu \mathrm{g} / \mathrm{mL}])$, and soaring interleukin-2 levels $(11,900 \mathrm{U} / \mathrm{mL}$ [reference range 158-623 $\mathrm{U} / \mathrm{mL}$ ]). The fibrinogen level was within normal limits.

Summating the entire clinical scenario, the diagnosis of immunodeficiency and HLH was made. Aggressive antibiotic and antifungal therapy continued, and HLH protocol was not initiated at the first instance. Within a week, defervescence was achieved with recession of inflammatory markers. Whole-exome sequencing then reported with a homozygous pathogenic variant $c .229 C>T(p$. $R 77 X$ ) in the NCF-2 gene, with no concurrent primary HLH mutations. Both parents were heterozygous carriers of the aforementioned mutation.

Thus, the diagnosis of an autosomal recessively inherited CGD with secondary HLH was confirmed. The patient was then repatriated on prophylactic antibiotic therapy and a strong recommendation for potential bone marrow transplantation. Her course thereafter remains unknown as she was lost to follow-up.

\section{Discussion}

Our case demonstrates the classic clinical course of an undiagnosed immunodeficient child, with the additional unique aspect of an autosomal recessively inherited, rarer form of CGD due to NCF-2 gene mutation. Recognition of the NCF-2 gene mutation holds added clinical relevance in the context of parental consanguinity with a history of multiple miscarriages and bears importance for genetic counseling.

The association between HLH and CGD has been well established, yet often proves itself as a clinical dilemma management wise. HLH is immune hyperactivity response due to impairment of natural killer and cytotoxic T-cell functioning [11]. Clinical severity ranges from a mild spontaneously resolving form to severe systemic inflammation, multiorgan failure, and death [11]. In reference to HLH-2004 diagnostic criteria, our patient had displayed five out of the eight features, namely, fever, splenomegaly, hyperferritinemia, hypertriglyceridemia, and raised interleukin-2 receptor levels. The diagnosis of infection-associated hemophagocytic syndrome can be made, supported by absence of primary HLH mutations on whole-exome sequencing. Where clinicians may attribute persistence of fever or clinical sepsis solely to CGD, it is crucial to bear secondary HLH in mind. Treatment of infection-associated hemophagocytic syndrome requires balance between infection control and immune suppression, and in some cases, early initiation of HLH protocol may be superfluous, if not also harmful. Our case demonstrates the value of aggressive antimicrobial therapy in controlling the progression of HLH.

CGD with Secondary Hemophagocytic Lymphohistiocytosis

\section{Conclusion}

CGD presents as recurrent severe infections and aberrant systemic granuloma formation. A positive family history of early deaths due to infectious causes is a particular red flag, even more so in offspring of consanguineous parents. Cases of CGD with persistent fever and raised inflammatory markers should raise suspicion for $\mathrm{HLH}$ in addition to a likely severe infection. The risks of immunosuppression and overwhelming sepsis may be averted with implementation of aggressive antimicrobial therapy in early stages of secondary HLH.

\section{Statement of Ethics}

Ethical approval was not required for this study in accordance with the Dubai Health Authority Research Committee policies. Written informed consent was obtained from the patient's parents for publishing the case report including any accompanying materials.

\section{Conflict of Interest Statement}

The authors have no conflicts of interest to declare.

\section{Funding Sources}

The authors have no funding sources to declare.

\section{Author Contributions}

All authors have contributed to writing, researching, and finalizing the case report.

\section{Data Availability Statement}

All data generated or analyzed during this case are included in this article. Further enquiries can be directed to the corresponding author.
References
1 Leiding JW, Holland SM. Chronic granulo- matous disease. In: Adam MP, Ardinger HH, Pagon RA, Wallace SE, Bean LJH, Gripp KW, et al., editors. GeneReviews ${ }^{\oplus}$ [Internet]. Seat- tle, WA: University of Washington; 1993- 20212012 Aug 9 [updated 2016 Feb 11].
2 Roos D, de Boer M. Molecular diagnosis of chronic granulomatous disease. Clin Exp Im- munol. 2014;175(2):139-49.
3 Schneider A, Ranza E, Diana A, Laurent M, Rougemont A, Hauri M, et al. A 19-month- old boy with refractory cervical adenitis. Pe- diatr Infect Dis J. 2019 Oct;38(10):1071. 
4 Saleem N, Ahmed TA, Bashir M. Chronic granulomatous disease. J Pak Med Assoc. 2016 Jan;66(1):97-100. Department of Immunology, Armed Forces Institute of Pathology, Rawalpindi, Shifa International Hospital, Islamabad.

5 Teimourian S, Sazgara F, de Boer M, van Leeuwen K, Roos D, Lashkary S, et al. Characterization of 4 new mutations in the CYBB gene in 10 Iranian families with $\mathrm{x}$ linked chronic granulomatous disease. J Pediatr Hematol Oncol. 2018;40(5):e26872.

6 Kilvert M, Roberts A, Hildebrand KJ. Case 3: persistent fever in an 8-month-old boy. Pediatr Rev. 2017 Mar;38(3):141.
7 Vignesh P, Bhattad S, Shandilya JK, Vyas S, Garg R, Rawat A. Vertebral osteomyelitis and acinetobacter Spp. Paravertebral soft tissue infection in a 4-year-old boy with X-linked chronic granulomatous disease. Pediatr Infect Dis J. 2016 Sep;35(9):1043-5.

8 Vignesh P, Vignesh P, Rawat A, Rawat A, Kumar A, Kumar A, et al. Chronic granulomatous disease due to neutrophil cytosolic factor (NCF2) gene mutations in three unrelated families. J Clin Immunol. 2017 Feb;37(2): 109-12.
$9 \mathrm{Wu}$ J, Wang W, Zhang Y, Chen T. Clinical features and genetic analysis of 48 patients with chronic granulomatous disease in a Single Center Study from Shanghai, China (2005-2015): New Studies and a literature review. J Immunol Res. 2017 Jan 30;2017: 8745254-17.

10 Morimoto A, Nakazawa Y, Ishii E. Hemophagocytic lymphohistiocytosis: pathogenesis, diagnosis, and management. Pediatr Int. 2016 Sep;58(9):817-25.

11 Parekh C, Hofstra T, Church JA, Coates TD. Hemophagocytic lymphohistiocytosis in children with chronic granulomatous disease. Pediatr Blood Cancer. 2011 Mar;56(3): $460-2$. 